\title{
The complex structural mechanisms behind strain curves in bismuth sodium titanate- barium titanate
}

Cite as: Appl. Phys. Lett. 116, 182902 (2020); https://doi.org/10.1063/5.0005401

Submitted: 21 February 2020 . Accepted: 19 April 2020 . Published Online: 04 May 2020

Kai-Yang Lee (D), Xi Shi, Nitish Kumar, Mark Hoffman (D), Martin Etter (D), Jens Winter, Lucas Lemos da Silva (D), Daniela Seifert, and Manuel Hinterstein (D)
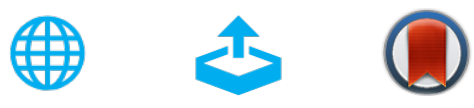

View Online

Export Citation

CrossMark

\section{ARTICLES YOU MAY BE INTERESTED IN}

Dense ferroelectric-ferroelastic domain structures in rhombohedral PMN-28PT single crystals Applied Physics Letters 116, 182901 (2020); https://doi.org/10.1063/5.0008522

Nanodomain patterns in ultra-tetragonal lead titanate $\left(\mathrm{PbTiO}_{3}\right)$

Applied Physics Letters 116, 182903 (2020); https://doi.org/10.1063/5.0007148

$\mathrm{BaTiO}_{3}$-based piezoelectrics: Fundamentals, current status, and perspectives Applied Physics Reviews 4, 041305 (2017); https://doi.org/10.1063/1.4990046

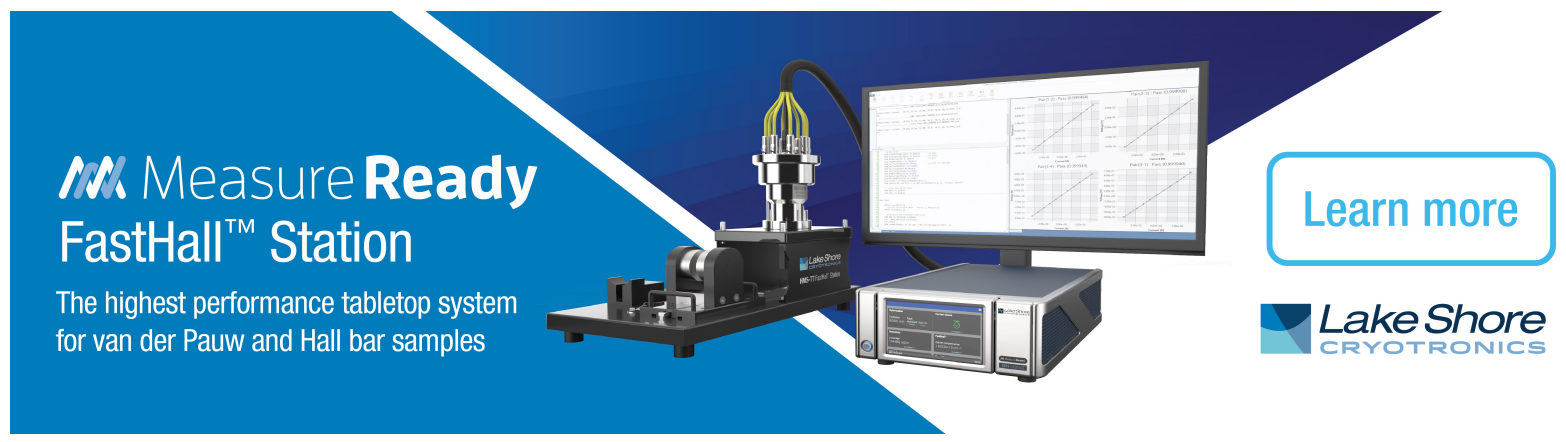




\title{
The complex structural mechanisms behind strain curves in bismuth sodium titanate-barium titanate
}

\author{
Cite as: Appl. Phys. Lett. 116, 182902 (2020); doi: 10.1063/5.0005401 \\ Submitted: 21 February 2020 - Accepted: 19 April 2020 • \\ Published Online: 4 May 2020
}
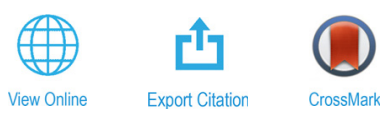

Kai-Yang Lee,, ${ }^{1, a)}$ (D) Xi Shi, ${ }^{2}$ Nitish Kumar, ${ }^{2}$ Mark Hoffman, ${ }^{2}$ (D) Martin Etter, ${ }^{3}$ (D) Jens Winter, ${ }^{4}$ Lucas Lemos da Silva, ${ }^{7}$ (iD Daniela Seifert, ${ }^{1}$ and Manuel Hinterstein ${ }^{1,2}$ (iD

\author{
AFFILIATIONS \\ ${ }^{1}$ Institute for Applied Materials, Karlsruhe Institute of Technology, 76131 Karlsruhe, Germany \\ ${ }^{2}$ School of Materials Science and Engineering, UNSW Sydney, 2052 Sydney, Australia \\ ${ }^{3}$ Deutsches Elektronensynchrotron DESY, 22607 Hamburg, Germany \\ ${ }^{4}$ Department of Physics, University of Siegen, 57068 Siegen, Germany
}

a) Author to whom correspondence should be addressed: kai-yang.lee@kit.edu

\begin{abstract}
In this work, the lead-free composition $(1-x) \mathrm{Bi}_{0.5} \mathrm{Na}_{0.5} \mathrm{TiO}_{3}-x \mathrm{BaTiO}_{3}(\mathrm{BNT}-\mathrm{BT})$ with $x=0.12$ was investigated using in situ Synchrotron $\mathrm{x}-$ ray powder diffraction. With the applied electric field, the pseudo-cubic relaxor phase reversibly transforms to a ferroelectric state. The reversibility is still preserved after $10^{4}$ bipolar electric field cycles. A Rietveld refinement with a structure, strain, and texture analysis using a model based on the atomic scale was applied for four frequencies from $10^{-4}$ to $10^{1} \mathrm{~Hz}$. The analysis allowed us to separately determine the two coexisting phases, their electric field dependent evolution, and the underlying strain mechanisms. For all the applied frequencies, we showed that domain switching is the only strain mechanism appearing in the tetragonal phase and the lattice strain is the only mechanism in the rhombohedral phase. The coercive field of the tetragonal phase $(4 \mathrm{kV} / \mathrm{mm})$ is found to be higher than that of the rhombohedral phase $(3 \mathrm{kV} / \mathrm{mm})$. This divergence has not been observed in previously investigated lead-containing materials and cannot be detected solely using macroscopic strain and polarization experiments. Moreover, the domain strain abruptly starts to occur only after a threshold field value and exhibits high hysteresis. The lattice strain, on the other hand, starts nearly from the beginning and increases more linearly during the bipolar field cycle. It could, therefore, be demonstrated that complex structural mechanisms underlie the apparent clear and continuous macroscopic strain curve. These findings are crucial for all actuator materials undergoing a relaxor to ferroelectric phase transformation and provide approaches and strategies to optimize lead-free materials for tailored applications.
\end{abstract}

Published under license by AIP Publishing. https://doi.org/10.1063/5.0005401

The ability of piezoelectric materials to couple mechanical and electrical energies enables a wide range of applications mainly for actuator, sensor, motor, and transducer technologies. ${ }^{1,2}$ Due to the toxicity of lead-based materials, the European Union (EU) initiated legislations that rapidly sparked the research on lead-free alternative materials in recent years.

Specifically, $(1-x) \mathrm{Bi}_{0.5} \mathrm{Na}_{0.5} \mathrm{TiO}_{3}-x \mathrm{BaTiO}_{3}(\mathrm{BNT}-x \mathrm{BT})$ is in the focus of interest due to the high field-induced strain and a comparatively high electromechanical coupling factor $\left(\mathrm{k}_{\mathrm{p}}=20 \%\right) .{ }^{5,6}$ Likewise in the lead-based material lead zirconate titanate (PZT), the high strain is found at a morphotropic phase boundary (MPB), located at compositions of around $x=0.06-0.07 .7,8$ Apart from the region of the MPB, auspicious properties have also been discovered in BT-rich $(>11 \%)$ compositions, particularly PIC 700 (PI Ceramics, Lederhose,
Germany) as an example of an already commercially available BT-rich material. $^{9,10}$

Even though these BT-rich compositions are far away from the MPB with two coexisting phases, ${ }^{11}$ a phase transformation can still be induced due to the changing properties in a non-stoichiometric material. $^{12}$ The long-ranged ferroelectricity (FE) is evoked by the application of a sufficiently large electric field, which transforms back into a relaxor (R) state once the field is lowered. ${ }^{12}$ This reversible phase transformation is typically responsible for giant strain, previously shown in $\quad 0.02 \mathrm{Bi}_{0.5} \mathrm{Na}_{0.5} \mathrm{TiO}_{3}-0.06 \mathrm{BaTiO}_{3}-0.02\left(\mathrm{~K}_{0.5} \mathrm{Na}_{0.5}\right) \mathrm{NbO}_{3}$ (BNT-BT-KNN) and manganese-doped BNT-BT. ${ }^{13}$

Additionally, Li et al. showed that stoichiometric deviations in BNT lead to significant differences in the microstructural and electrical properties. ${ }^{16,17}$ Introducing a non-stoichiometry, by increasing the 
$\mathrm{Bi} / \mathrm{Na}$ ratio in BNT-BT, stabilizes the relaxor state at room temperature in favor of the tetragonal ferroelectric phase, which is expected in stoichiometric compositions based on the previously suggested phase diagrams. $^{12,18-20}$

Although a large macroscopic strain can be achieved, the underlying structural effects and mechanisms in the bulk material remain unknown. For that, in situ synchrotron x-ray diffraction with data analysis using the STRAP (Strain, Texture and Rietveld Analysis for Piezoceramics) procedure ${ }^{21}$ is utilized (see the supplementary material for Rietveld refinement details). Here, an orientation series with specific geometries can differentiate all strain mechanisms for all the coexisting phases of the material with a single experiment. In previous works with both PZT and lead-free materials, this approach was already performed to quantify different strain mechanisms for each phase separately to reconstruct the field-induced macroscopic strain response. ${ }^{11,21-27}$ Generally, the strain mechanisms are known as the domain switching effect, caused by extrinsic domain wall motions, and second the converse piezoelectric effect (lattice strain), which is ascribed to intrinsic unit cell expansions. The lattice strain occurring in the tetragonal phase can be detected by shifting the 111 reflection, whereas non- $180^{\circ}$-domain-switching effects lead to texture effects that are discernible in 200 reflection intensity ratios. ${ }^{25,28}$ One of the basic assumptions made in the STRAP approach is the summation of lattice and domain strain, which is well established since the work of Hall et al. has been published. ${ }^{28}$ Since the two different strain mechanisms show clearly separable effects in the diffraction patterns, the STRAP method allows a comprehensive interpretation.

Another important aspect regarding the functionality of piezoelectric materials especially in actuator applications is the frequency of the applied electric field. The frequency determines the kinetics of the sample and therefore poses a challenge for the performance of a stable strain. Previous works investigating both lead and lead-free compositions suggested a self-heating of the sample caused by higher frequencies. ${ }^{12,29-32}$ At elevated temperatures, the necessary threshold electric field for the R-FE phase transformation was also found to increase. ${ }^{30}$ Shi et al. presented an electric field-temperature $(E-T)$ phase diagram of BNT-12BT, based on temperature dependent permittivity and piezoelectric measurements. A sharp increase occurs within a temperature change of $\sim 10^{\circ} \mathrm{C}$ only, which shows the large impact of the temperature on the phase transformation behaviour. ${ }^{20}$ It is, therefore, crucial to gain greater insight into the often overlooked frequency dependence since actuator materials require a stable strain over a broad range of frequencies in real working conditions.

In the present work, BNT-12BT with a non-stoichiometrically increased $\mathrm{Bi} / \mathrm{Na}$ ratio in the $\mathrm{BT}>11 \%$ composition range is investigated using STRAP in order to elucidate the structural strain mechanisms. This enables a comprehensive structural approach of lead-free piezoceramic strain mechanisms, based on experiments in a broad range of frequencies. The calculated phase combined strain curves are compared with the macroscopically acquired strain data. The findings are relevant not only for BNT-BT specifically but also for enhancing the understanding of all materials exhibiting a giant strain, caused by a reversible R-FE transformation. This work, based on quantitative methods, offers an unprecedented insight into the underlying strain effects and features key knowledge for future research.

A $0.88 \mathrm{Bi}_{0.53} \mathrm{Na}_{0.47} \mathrm{TiO}_{3}-0.12 \mathrm{BaTiO}_{3}$ sample of dimensions 0.97 $\times 1.12 \times 5.11 \mathrm{~mm}^{3}$ was investigated in the present study, whose detailed stoichiometry determination, processing parameters, and microstructure investigations are given elsewhere. ${ }^{1,33}$ The $\mathrm{x}$-ray diffraction experiments were performed at beamline P02.1 at the Deutsches Elektronen-Synchrotron (DESY) in Hamburg, Germany. ${ }^{34,35}$ High-energy $\mathrm{x}$-rays with a photon energy of $60 \mathrm{keV}$ and a wavelength of $0.20718 \mathrm{~A}$ were used. To investigate bulk properties, all experiments were performed in transmission geometry using a tailored sample environment setup. ${ }^{12}$ The detecting system consists of a twodimensional (2D) flat panel detector XRD $1621 \mathrm{~N}$ ES Series (PerkinElmer), with 16-in. and $2048 \times 2048$ pixels of the size of $200 \mu \mathrm{m}$ each. A bipolar triangular electric field cycle with an amplitude of $5 \mathrm{kV} / \mathrm{mm}$ was applied to the painted silver electrodes on two opposing parallel sides of the sample. The applied field frequencies are 1.9 $\times 10^{-4} \mathrm{~Hz}$ with an exposure time of $30 \mathrm{~s}, 10^{-3} \mathrm{~Hz}$ with an exposure time of $10 \mathrm{~s}$, and $5 \times 10^{-2} \mathrm{~Hz}$ with an exposure time of $0.2 \mathrm{~s}$ for each diffraction image. To obtain images with data of sufficient statistic quality for the fourth frequency at $10 \mathrm{~Hz}$, a $1 \mathrm{D}$ multi-analyser point detector (MAD) in stroboscopic mode was utilized instead of the 2D detector. $^{36,37}$ While the 2D detecting system allows collecting data from the complete diffracted Debye rings for orientation-dependent analysis, a 1D detector acquires only one specific azimuthal line of the Debye rings. To enable data acquisition of different orientations, three different orientation angles of $0^{\circ}, 45^{\circ}$, and $90^{\circ}$ were set for the measurement. Every single 2D diffraction image during the field cycle was divided into 18 equally sized $5^{\circ}$-slices, and each was integrated using the software Fit2D. Requiring quantitatively correct values for the subsequent STRAP procedure, the beam absorption needed to be calculated, taken into account the beam setup, sample geometry, and the sample stoichiometry. The detailed derivation of the absorption correction is provided in the supplementary material.

The 18 resulting diffraction patterns were analyzed by the STRAP method, using the software package Material Analysis Using Diffraction (see the supplementary material for refinement details). To compare the results with the macroscopic measurements, a TF analyser (TF Analyzer 2000, aixACCT, Germany) was used for electromechanical characterisation.

A coexistence of tetragonal and rhombohedral phases results as the best structure model for an appropriate refinement. A full STRAP procedure for every electric field step yields the phase fractions [Fig. $1(a)]$ as well as the tetragonal unit cell parameters $a$ and $c$ and hence the tetragonal unit cell distortion $\eta_{T}$ [Fig. 1(b)], which is defined by $\eta_{T}=\left(\frac{c}{a}-1\right) \times 100$. The rhombohedral unit cell distortion $\eta_{R}$ is negligible for all field steps. Therefore, the rhombohedral phase can be regarded as a pseudo-cubic phase throughout the field cycle. Although a)

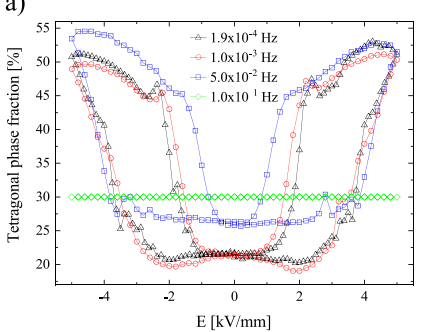

b)

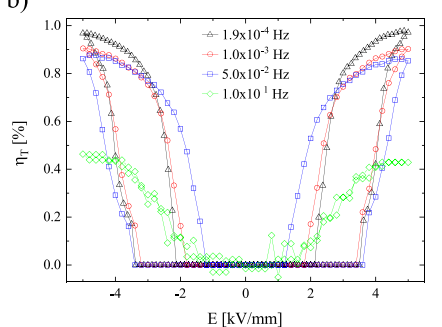

FIG. 1. The tetragonal phase fraction (a) and tetragonal unit cell distortion $\eta_{\mathrm{T}}$ (b), both measured at four different electric field frequencies. 
Fig. 1(a) shows phase fractions over the whole electric field cycle, the tetragonal unit cell distortion $\eta_{T}$ indicates that below a certain electric field, the tetragonal phase can also be regarded to be pseudo-cubic. This is evident from Fig. 1(b), in which the tetragonal lattice parameters are set pseudo-cubic with $\eta_{T}=0 \%$ for a range of lower field values. Therefore, the results indicate the transformation from a single pseudo-cubic phase to a phase coexistence of a distorted tetragonal phase and a pseudo-cubic rhombohedral phase, similar to observations in $\mathrm{Bi}_{0.5} \mathrm{Na}_{0.5} \mathrm{TiO}_{3}-\mathrm{SrTiO}_{3}{ }^{38}$

The plotted field-dependent unit cell distortions further demonstrate that no tetragonal distortion appears until a threshold electric field of around $3.5 \mathrm{kV} / \mathrm{mm}$ is reached. Above this value, a high tetragonal distortion precipitates for the frequencies of $1.9 \times 10^{-4}, 1 \times 10^{-3}$, and $5 \times 10^{-2} \mathrm{~Hz}$. This is in agreement with a reversible R-FE phase transformation behaviour. ${ }^{12}$ With decreasing frequencies, the backward FE-R transformation is delayed and the $\mathrm{R}$ state is reached at around $1 \mathrm{kV} / \mathrm{mm}$ instead of $2 \mathrm{kV} / \mathrm{mm}$ for lower frequencies. Applying the frequency of $5 \times 10^{-2} \mathrm{~Hz}$, the amplitude of phase transformation is also reduced from around $30 \%$ to around $25 \%$.

It should be noted that the tetragonal phase fraction measured at $10 \mathrm{~Hz}$ [Fig. 1(a), green] is fixed to $30 \%$ during the entire field cycle since the deviating values were negligibly small. The overall level of the tetragonal phase fractions shifts to higher levels and remains even in the remanent state at an increased value. This and the effect of a more pronounced hysteresis indicate that kinetic effects lead to delayed responses at slightly higher frequencies.

It can be concluded that the formation of the tetragonally distorted phase requires long reaction times and occurs abruptly after reaching the threshold field value. Additionally, the tetragonal lattice distortion $\eta_{T}$ [Fig. 1(b)] decreases with increasing frequencies. The distortions at $10 \mathrm{~Hz}$ reach only half the values compared to the lowest frequency and appear to be more saturated. This indicates that kinetic effects not only affect the tetragonal phase formation but also the lattice distortion of the FE phase. The hysteresis behavior of the tetragonal phase distortion is analogous to that of the phase fractions. At $10 \mathrm{~Hz}$, although the frequency is further increased by a factor of 10 , the hysteresis vanishes, so that unit cell distortion processes already start around $1.5 \mathrm{kV} / \mathrm{mm}$. The appearance of this counteracting effect is possibly owing to a higher domain mobility due to the self-heating effect that shifts the temperature of the sample near the depolarization temperature. ${ }^{12}$

Applying two different models for texture and strain analyses, the two phases can be regarded separately with respect to the two different strain mechanisms. The STRAP method was performed to quantify the different strain contributions in the tetragonal [Fig. 2(a)], the rhombohedral [Fig. 2(b)], and the resulting phase weighted overall strain [Fig. 2(c)] (see the supplementary material for calculation details). In comparison to the calculated curves, Fig. 2(d) depicts the macroscopic measured strain curves at field frequencies in the range of $10^{-4}-10^{1} \mathrm{~Hz}$.

During the refinements, no lattice strain could be refined for the tetragonal phase. Since no unit cell distortion is observed with the highest possible resolution as well, no domain switching strain could be refined for the rhombohedral phase. Hence, the tetragonal phase contributes only to domain switching strain $S_{D, T}$ [Fig. 2(a)] and the rhombohedral phase only to lattice strain $S_{L, R}$ [Fig. 2(b)]. This differentiation enables the observation that $S_{D, T}$ and $S_{L, R}$ mechanisms
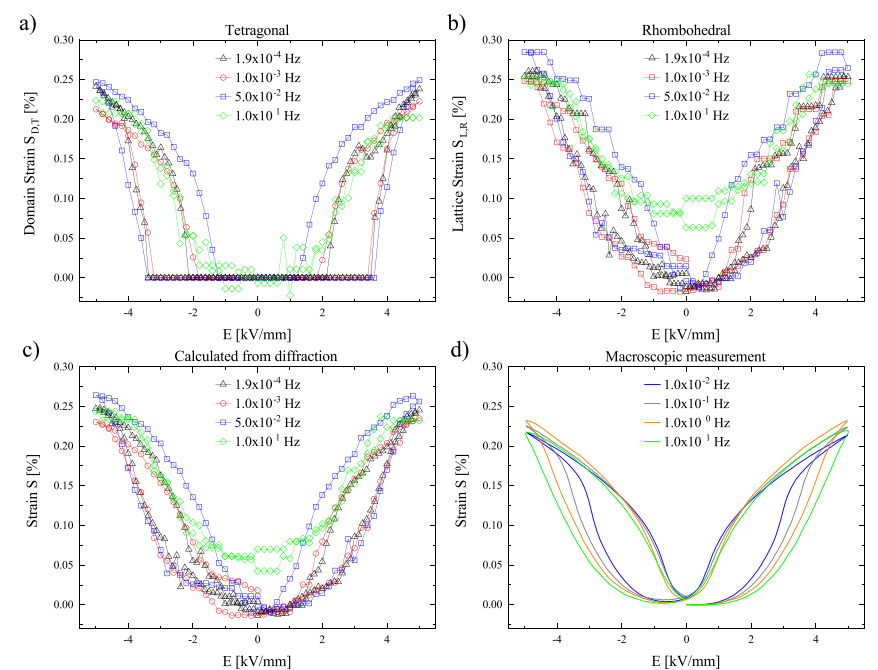

FIG. 2. Calculated tetragonal (a), rhombohedral (b), phase weighted combined (c), and macroscopic (d) strain curves of one bipolar electric field cycle at four different frequencies (colored lines).

exhibit a significantly different coercive field, which are around $3 \mathrm{kV} /$ $\mathrm{mm}$ for $S_{L, R}$ and $4 \mathrm{kV} / \mathrm{mm}$ for $S_{D, T}$. This is an outstanding difference from the behavior of lead-containing materials like PZT, where all strain mechanisms have the same coercive field. ${ }^{21}$ They might be the reason for the different behavior of lead-free and lead-containing piezoceramics. The phase weighted (with $F_{T}$ and $F_{R}$ ) combined strain $S$ [Fig. 2(c)] is, therefore, calculated with $S=F_{T} \times S_{D, T}+F_{R} \times S_{L, R} \cdot{ }^{25}$

The domain strain response in the tetragonal phase at 1.9 $\times 10^{-4}, 1 \times 10^{-3}$, and $5 \times 10^{-2} \mathrm{~Hz}$ is negligibly small due to the nonexisting lattice distortion until about $3.5 \mathrm{kV} / \mathrm{mm}$. Exceeding this threshold field amplitude, the domain strain sharply increases, reaching a maximum strain value of $0.25 \%$. By lowering the field, the domain strain declines with a strong hysteresis to zero strain at $1 \mathrm{kV} / \mathrm{mm}$ for $5 \times 10^{-2} \mathrm{~Hz}$ and at $2 \mathrm{kV} / \mathrm{mm}$ in the case of the other two frequencies $1 \times 10^{-3}$ and $1.9 \times 10^{-4} \mathrm{~Hz}$. The domain strain response, including its hysteresis during the field cycle, coincides with the tetragonal phase fraction [Fig. 1(a)] and the tetragonal lattice distortion curves [Fig. 1(b)]. The simultaneous formation and parallel development of these effects, therefore, indicate a correlated process, which is triggered after reaching a certain threshold field value. The growth of domain strain can, therefore, be regarded to be dormant before a sufficient activation energy is provided. Also with the tetragonal phase distortion at $10 \mathrm{~Hz}$ [Fig. 1(b)], the accompanying strain curve (Fig. 2, green) exhibits no hysteresis for both $S_{D, T}$ and $S_{L, R}$. The frequency driven self-heating effect also leads to incipient domain switching processes already around $1.5 \mathrm{kV} / \mathrm{mm}$. Nevertheless, a remanent strain due to slower electric field dependent responses can be located around $0.075-0.1 \%$.

Regarding the shape of the $S_{L, R}$ curves, fundamental differences are discernible compared to $S_{D, T}$. The strain emerges rather continuous and gradually, starting already from 0 to $1 \mathrm{kV} / \mathrm{mm}$. The hysteresis is less pronounced and more constant than that for $S_{D, T}$ during all the field steps. For both $S_{D, T}$ and $S_{L, R}$, the strain curves of all frequencies except $10 \mathrm{~Hz}$ coincide with the increasing field, but a clear difference is 
visible between $5 \times 10^{-2} \mathrm{~Hz}$ and the other frequencies upon reversing the field, which leads to different hystereses. However, for $S_{L, R}$, the differences with the reversed field are less pronounced.

The following process can, therefore, be concluded sequentially for $1 \times 10^{-3} \mathrm{~Hz}$ : At low electric fields $(0-1 \mathrm{kV} / \mathrm{mm})$, no strain response is present. Between 1 and $2 \mathrm{kV} / \mathrm{mm}$, only $S_{L, R}$ appears, until $3.5 \mathrm{kV} / \mathrm{mm}$, where the domain strain also responds with a sudden sharp rise. Afterwards, $S_{D, T}$ and $S_{L, R}$ increase simultaneously, whereas $S_{D, T}$ responds more sharply and more linearly. Upon reversing the electric field, the shape of the curve remains the same, except for a reduced slope.

For the calculated curves, an increasing strain hysteresis for higher frequencies can be clearly observed, which is in agreement with the kinetic effects described above (Fig. 1). However, the hysteresis of the macroscopic curve at $10 \mathrm{~Hz}$ continues to increase in contrast to the calculated strain curve. This can be explained by the experimental setup, where large metal contacts, fixing the sample, dissipate the selfgenerated heat and therefore impede the described temperature effect.

Furthermore, for lower frequencies, the hysteresis narrows toward higher field values, which coincides with the calculated strain curves. Compared to the curves obtained from macroscopic measurements, the calculated combined strain curves exhibit a slightly larger maximum strain value. The reason possibly lies in the mechanical limitations due to the pressure on the sample exerted by the metal contacts that are used in the macroscopic strain measurement setup. The pressure also explains the increasing coercive field at higher frequencies while increasing the field, whereas the strain inflection points coincide by field reversal [Fig. 2(d)]. This behavior demonstrates the impact of the blocking force that quantifies the force under which a displacement of the sample is completely blocked. This is an especially relevant characteristic for evaluating actuator materials in operation. ${ }^{39,40}$ Even though the blocking force in purely ferroelectric BNT is higher than that in lead containing materials, an influence of the applied stress is discernible. ${ }^{41,42}$ This indicates that the blocking force for materials undergoing a field-induced phase transformation may be significantly lower than that for single-phase materials with a solely ferroelectric domain switching mechanism.

This effect is not observed in the calculated strain curves [Fig. 2(c)], where the experimental setup allowed a freestanding sample under zero-stress conditions. Here, the strain curves differ only upon field reversal since no pressure is present to favor the domain reorientation. Apart from these differences, the shapes of the calculated strain curves are in agreement with those of the macroscopic measurements, which indicates the suitability of the STRAP method. Although the overall strain curves appear rather simple, the results in this work illustrate the complex strain mechanisms that contribute to this behavior.

It has been shown that the structural approach to infer the overall strain from structural mechanisms represents a unique opportunity to quantify the strain in piezoceramics. Particularly, the nonstoichiometric and lead-free material BNT-BT in the BT $>11 \%$ composition range is interesting for its promising properties and the additional feature of the reversible R-FE phase transformation.

Based on models on atomic scales, structural strain and texture analyses of the $\mathrm{x}$-ray data reveal the coexisting rhombohedral and tetragonal phases. Herein, several differences arise regarding the behavior of the field-induced strain mechanisms. First, the coercive field in the case of the tetragonal phase is higher than that in the rhombohedral phase, which is not discernible from macroscopic measurements. Second, the strain of the rhombohedral phase is due to intrinsic lattice strain only, while the strain of the tetragonal phase consists merely of domain switching mechanisms. Third, the domain switching strain rises sharply at specific field thresholds and declines at different field values, depending on the applied frequencies. It can be concluded that the formation of the distorted tetragonal phase is a comparatively sluggish effect, which is abruptly accelerated by exerting domain strain upon reaching the threshold field value of $3.5 \mathrm{kV} / \mathrm{mm}$. The lattice strain occurring in the rhombohedral phase, on the other hand, starts in an early stage and increases rather continuous. Furthermore, a larger hysteresis effect is found in the domain strain curves, which also further increases by applying higher frequencies. This shows a relatively long reaction time of the distortion in the tetragonal phase responsible for domain strain. However, a contrary effect was found at the highest applied frequency of $10 \mathrm{~Hz}$, where no hysteresis appeared due to the effect of self-heating in the sample. These findings indicate the significant impact of the applied frequencies on the kinetics and therefore on the performance of the material.

Thus, even though the macroscopically measured strain curves exhibit a continuous behavior and suggest a plain mechanism at first glance, the underlying structural and microstructural mechanisms can be rather complex. This work provides detailed information about the relation of macroscopic and structural electric field-induced mechanisms, which is worthwhile for all materials exhibiting R-FE transformations. It is also important to underline that the underlying interplay of the strain mechanisms is fundamentally different from that in PZT and lead-based piezoceramics. This knowledge opens up possibilities to precisely tackle the requirements and enables target-oriented amendments for specific applications in lead-free materials. This approach not only effectively contributes to the development of future materials but also particularly stimulates further work to push the commercialization of lead-free alternative piezoceramic systems.

See the supplementary material for the derivation of the absorption correction, the detailed Rietveld refinement procedure, and the strain calculation.

The authors would like to thank the German Research Society (Deutsche Forschungsgemeinschaft, DFG) for Grant No. HI 1867/ 1-1. The beamtime at beamline P02.1 at the Deutsches Elektronensynchrotron (DESY, Hamburg, Germany) is greatly acknowledged.

The data that support the findings of this study are available within this article and its supplementary material.

\section{REFERENCES}

'J. Rödel, W. Jo, K. T. P. Seifert, E. M. Anton, T. Granzow, and D. Damjanovic, Am. Ceram. Soc. 92, 1153 (2009).

${ }^{2}$ W. Jo, J. E. Daniels, J. L. Jones, X. Tan, P. A. Thomas, D. Damjanovic, and J. Rödel, J. Appl. Phys. 109, 014110 (2011).

${ }^{3}$ T. Abraham, "Piezoelectric ceramic, polymer and ceramic/polymer composite devices-Types, materials, applications, new developments, industry structure and global markets," presented at Innovative Research and Products, Inc. Stamford, 2014, Report Code ET-117, 319 pp., see http://www.innoresearch.net/ toc.aspx?id=ET-117.

${ }^{4}$ A. J. Bell and O. Deubzer, MRS Bull. 43, 581 (2018).

${ }^{5}$ E. Aksel and J. L. Jones, Sensors 10, 1935 (2010). 
${ }^{6}$ M. Hinterstein, M. Knapp, M. Hölzel, W. Jo, A. Cervellino, H. Ehrenberg, and H. Fuess, J. Appl. Crystallogr. 43, 1314 (2010).

${ }^{7}$ T. Takenaka, K. Maruyama, and K. Sakata, Jpn. J. Appl. Phys., Part 1 30, 2236 (1991).

${ }^{8}$ Z. Zhou, J. Luo, W. Sun, and J. F. Li, Phys. Chem. Chem. Phys. 19, 19992 (2017).

${ }^{9}$ P. Ditas, E. Hennig, A. Kynast, and P. I. C. Gmbh, Sens. Mess. Syst. 5, 5-8 (2014).

${ }^{10}$ C. H. Hong, H. P. Kim, B. Y. Choi, H. S. Han, J. S. Son, C. W. Ahn, and W. Jo, J. Mater. 2(1), 1 (2016).

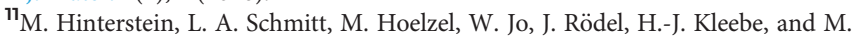
Hoffman, Appl. Phys. Lett. 106, 222904 (2015).

${ }^{12}$ K.-Y. Lee, X. Shi, N. Kumar, M. Ho, M. Etter, S. Checchia, J. Winter, L. Lemos, D. Seifert, M. Hinterstein, M. Hoffman, M. Etter, S. Checchia, J. Winter, L. Lemos da Silva, D. Seifert, and M. Hinterstein, Materials 13, 1054 (2020).

${ }^{13}$ W. Jo, T. Granzow, E. Aulbach, J. Rödel, and D. Damjanovic, J. Appl. Phys. 105, 094102 (2009).

${ }^{14}$ S. T. Zhang, A. B. Kounga, E. Aulbach, H. Ehrenberg, and J. Rödel, Appl. Phys. Lett. 91, 112906 (2007).

${ }^{15}$ E. Sapper, N. Novak, W. Jo, T. Granzow, and J. Rödel, J. Appl. Phys. 115, 194104 (2014).

${ }^{16}$ M. Li, M. J. Pietrowski, R. A. De Souza, H. Zhang, I. M. Reaney, S. N. Cook, J. A. Kilner, and D. C. Sinclair, Nat. Mater. 13, 31 (2014).

${ }^{17}$ M. Li, H. Zhang, S. N. Cook, L. Li, J. A. Kilner, I. M. Reaney, and D. C. Sinclair, Chem. Mater. 27, 629 (2015).

${ }^{18} \mathrm{C}$. Ma and X. Tan, J. Am. Ceram. Soc. 94, 4040 (2011).

${ }^{19}$ C. Ma, X. Tan, E. Dul'kin, and M. Roth, J. Appl. Phys. 108, 104105 (2010).

${ }^{20}$ X. Shi, N. Kumar, and M. Hoffman, J. Mater. Chem. C 6, 12224 (2018).

${ }^{21}$ M. Hinterstein, K.-Y. Lee, J. Glaum, A. J. Studer, M. Hoffman, and M. J. Hoffmann, Phys. Rev. B 99, 174107 (2019).

${ }^{22}$ S. Eßlinger, P. Neumeister, A. Schönecker, M. Hoffman, A. Studer, and M. Hinterstein, Adv. Eng. Mater. 21, 1900159 (2019).

${ }^{23}$ J. Glaum, Y. Heo, M. Acosta, P. Sharma, J. Seidel, and M. Hinterstein, Phys. Rev. Mater. 3, 054406 (2019).

${ }^{24}$ H. E. Mgbemere, G. A. Schneider, L. A. Schmitt, and M. Hinterstein, J. Ceram. Sci. Technol. 8, 45 (2017).

${ }^{25}$ M. Hinterstein, M. Hoelzel, J. Rouquette, J. Haines, J. Glaum, H. Kungl, and M. Hoffman, Acta Mater. 94, 319 (2015).
${ }^{26}$ N. H. Khansur, M. Hinterstein, Z. Wang, C. Groh, W. Jo, and J. E. Daniels, Appl. Phys. Lett. 107, 242902 (2015).

${ }^{27}$ Z. Wang, K. G. Webber, J. M. Hudspeth, M. Hinterstein, and J. E. Daniels, Appl. Phys. Lett. 105, 161903 (2014).

${ }^{28}$ D. A. Hall, A. Steuwer, B. Cherdhirunkorn, T. Mori, and P. J. Withers, J. Appl. Phys. 96, 4245 (2004).

${ }^{29} \mathrm{M}$. Acosta, W. Jo, and J. Rödel, J. Am. Ceram. Soc. 97, 1937 (2014).

${ }^{30}$ R. Dittmer, W. Jo, E. Aulbach, T. Granzow, and J. Rödel, J. Appl. Phys. 112, 014101 (2012)

${ }^{31}$ M. S. Senousy, R. K. N. D. N. D. Rajapakse, D. Mumford, and M. S. Gadala, Smart Mater. Struct. 18, 045008 (2009).

${ }^{32}$ P. Ronkanen, P. Kallio, M. Vilkko, and H. N. Koivo, "Self heating of piezoelectric actuators: Measurement and compensation," in Micro-Nanomechatronics and Human Science, 2004 and The Fourth Symposium MicroNanomechatronics for Information-Based Society, Nagoya, Japan, 2004 (IEEE, 2005), pp. 313-318.

${ }^{33}$ N. Kumar, X. Shi, and M. Hoffman, J. Eur. Ceram. Soc. 40, 2323 (2020).

${ }^{34}$ M. Herklotz, F. Scheiba, M. Hinterstein, K. Nikolowski, M. Knapp, A.-C. Dippel, L. Giebeler, J. Eckert, and H. Ehrenberg, J. Appl. Crystallogr. 46, 1117 (2013).

${ }^{35}$ A. Dippel, H. Liermann, J. T. Delitz, P. Walter, H. Schulte-Schrepping, O. H. Seeck, and H. Franz, J. Synchrotron Radiat. 22, 675 (2015).

${ }^{36}$ H. Choe, S. Gorfman, M. Hinterstein, M. Ziolkowski, M. Knapp, S. Heidbrink, M. Vogt, J. Bednarcik, A. Berghaeuser, H. Ehrenberg, and U. Pietsch, J. Appl. Crystallogr. 48, 970 (2015).

${ }^{37}$ H. Choe, S. Gorfman, S. Heidbrink, U. Pietsch, M. Vogt, J. Winter, and M. Ziolkowski, IEEE Trans. Nucl. Sci. 64, 1320 (2017).

${ }^{38}$ M. Acosta, L. A. Schmitt, C. Cazorla, A. Studer, A. Zintler, J. Glaum, H.-J. Kleebe, W. Donner, M. Hoffman, J. Rödel, and M. Hinterstein, Sci. Rep. 6, 28742 (2016).

${ }^{39}$ K. G. Webber, D. J. Franzbach, and J. Koruza, J. Am. Ceram. Soc. 97, 2842 (2014).

${ }^{40}$ L. Daniel, D. A. Hall, J. Koruza, K. G. Webber, A. King, and P. J. Withers, J. Appl. Phys. 117, 174104 (2015).

${ }^{41}$ G. Esteves, C. M. Fancher, S. Röhrig, G. A. Maier, J. L. Jones, and M. Deluca, Acta Mater. 132, 96 (2017).

${ }^{42}$ W. Jo, R. Dittmer, M. Acosta, J. Zang, C. Groh, E. Sapper, K. Wang, and J. Rödel, J. Electroceram. 29, 71 (2012). 